\title{
NOUVELLE
}

\section{La radiothérapie FLASH pour épargner les tissus sains}

Vincent Favaudon ${ }^{1}$, Charles Fouillade ${ }^{1}$, Marie-Catherine Vozenin ${ }^{2}$
${ }^{1}$ Inserm U 612, Institut Curie-Recherche, bâtiments 110-112, centre universitaire, 91405 Orsay, France ;

${ }^{2}$ Radio-oncologie/radiothérapie, centre hospitalier universitaire vaudois, 1011 Lausanne, Suisse.

vincent.favaudon@curie.fr marie-catherine.vozenin@chuv.ch

\section{Position du problème}

La radiothérapie est, avec la chirurgie, la technique la plus efficace pour le traitement des tumeurs solides. En France, près de 180000 patients bénéficient chaque année d'une radiothérapie à but curatif, palliatif ou symptomatique. Les progrès effectués durant les 30 dernières années ont été considérables et le taux de guérison, variable selon la localisation, le type et le stade des cancers, atteint aujourd'hui $60 \%$. Ces progrès sont liés pour l'essentiel à un gain en précision de l'imagerie, de la balistique et de la dosimétrie. Les diverses techniques de radiothérapie conformationnelle ont ainsi permis un meilleur contrôle local et une survie accrue. Toutefois, les technologies embarquées dans les accélérateurs linéaires d'électrons (canon, section accélératrice et production de rayons-X par freinage) utilisés en médecine et les modalités de délivrance de la dose, avec un débit de dose de l'ordre du Gray (Gy) par minute et une dose par fraction/jour de 2 Gy, n'ont guère évolué au cours des dernières décennies. D’autres procédés, comme l'irradiation par microfaisceaux (Pencil beam scanning, PBS) qui se développe en protonthérapie, mettent en œuvre des débits de dose instantanés qui peuvent atteindre $200 \mathrm{~Gy} / \mathrm{s}$, mais nécessitent un balayage du volume à irradier, de sorte que le temps nécessaire pour traiter une tumeur n'est pas très différent de celui qui prévaut en radiothérapie conventionnelle. Les réponses à ces nouvelles modalités de traitement en termes de complications (fibrose, cancers radio-induits, etc.) et d'efficacité antitumorale n'ont pas fait l'objet de recherches systématiques.
C'est cette lacune que nous avons cherché à combler en modifiant le débit de dose.

\section{Premiers indices}

Prempree et al. [1] avaient montré sur des cultures de cellules in vitro qu'une irradiation impulsionnelle (FLASH - dans ce cas précis 2 nanosecondes) produit moins d'aberrations chromosomiques d'échange qu'une irradiation conventionnelle (CONV - quelques minutes). Cette observation a été récemment confirmée par d'autres auteurs [2]. Nous mêmes avions montré [3, 4] qu'à dose égale, le taux de mort cellulaire différée, conséquence de l'instabilité chromosomique induite par l'irradiation, est considérablement plus faible après une irradiation FLASH qu'après une irradiation CONV. Or, à l'échelle d'un tissu, le mécanisme de mort cellulaire est un déterminant important des effets à long terme de l'irradiation, en particulier visà-vis de la survenue des complications.

Une méthodologie peu conventionnelle L'ensemble de ces observations nous ont conduits à étudier la fibrose pulmonaire radio-induite chez des souris (57BL/6) $[5,6]$ exposées en une seule fois à une dose de 17 Gy localisée au thorax en mode FLASH ( $\geq 60 \mathrm{~Gy} / \mathrm{s}$, durée d'irradiation $\leq 0,5 \mathrm{~s})$ et en mode CONV $(0,03 \mathrm{~Gy} / \mathrm{s}$, durée d'irradiation > $500 \mathrm{~s}$ ). Pour ces études, nous avons utilisé un accélérateur linéaire d'électrons, prototype qui fonctionne au sein du centre de recherche de I'Institut Curie à Orsay et délivre des électrons de 4,5 MeV avec un débit de dose moyen qui peut atteindre $2000 \mathrm{~Gy} / \mathrm{s}$. Les souris irradiées selon l'un ou l'autre mode ont été suivies pendant 36 semaines. Les poumons ont été prélevés à intervalles de huit semaines avec examen des animaux avant (poids, état de la peau) et après autopsie (état des organes situés dans ou hors du champ d'irradiation, recherche d'une éventuelle pneumonie au stade congestif, etc.). Les poumons ont été fixés et préparés en coupes sériées pour caractérisation histologique de l'existence d'une fibrose dont le stade a été analysé selon une échelle appropriée. D'autres échantillons ont été préparés pour l'analyse du taux d'apoptose ou de l'expression du TGF- $\beta$ (transforming growth factor). Le pouvoir antitumoral des deux modalités d'irradiation a ensuite été comparé, d'une part sur des xénogreffes de tumeurs humaines ( $\mathrm{HBCX}-12 \mathrm{~A}$, carcinome du sein triple négatif; $H \varepsilon p-2$, carcinome tête et cou) implantées chez la souris Swiss nude, d'autre part sur des tumeurs syngéniques orthotopiques (carcinome TC-l Luc+) localisées au poumon de souris (57BL/6).

L'irradiation FLASH protège le poumon [7] Les animaux exposés à 17 Gy CONV développent tous une alvéolite aiguë évoluant en 16 à 24 semaines en une fibrose pulmonaire complète. En revanche, aucune complication n'est observée chez les souris exposées à la même dose en mode FLASH. II faut monter à 30 Gy FLASH pour produire une fibrose histologiquement comparable à celle observée après 17 Gy CONV, et induire l'expression du TGF$\beta$, un marqueur universel de la fibrose pulmonaire [8]. Une réduction spectactulaire du taux d'apoptose radio-induite est observée au niveau des cellules musculaires lisses entourant les artérioles 


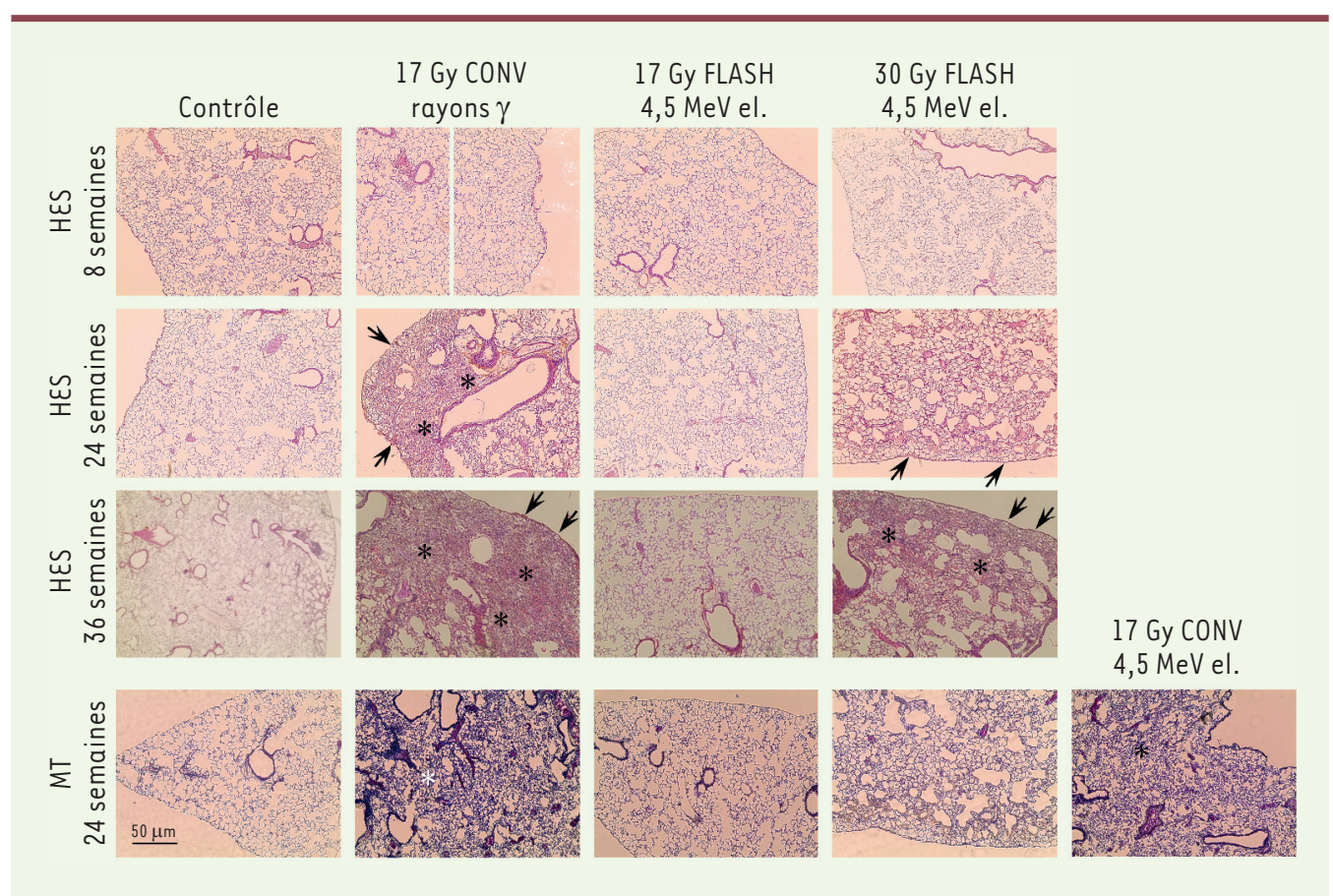

Figure 1. Analyse histologique de la fibrose pulmonaire radio-induite. Des souris (57BL/6) ont été exposées en dose unique à une irradiation du thorax bilatérale à débit de dose conventionnel (CONV : 0,03 Gy/s, rayons$\gamma$ ou électrons de 4,5 MeV) ou pulsé (FLASH : $60 \mathrm{~Gy} / \mathrm{s}$, électrons de 4,5 MeV). Les poumons ont été prélevés aux temps indiqués, fixés, coupés et colorés (HES : hématoxyline-éosinesafran; MT: trichrome Masson). 17 Gy CONV induisent en 24 semaines une fibrose massive avec épaississement des parois alvéolaires par le collagène, indifféremment de la source de rayonnement. Aucune lésion fibrotique n'est observée lorsque la même dose de 17 Gy est délivrée en mode FLASH. Une dose de 30 Gy FLASH est nécessaire pour induire le même niveau de fibrose que 17 Gy CONV. Les flèches et les astérisques indiquent les zones de fibrose sous-pleurale et intra-parenchymateuse, respectivement (avec la permission de Science Translational Medicine [7]).

et les bronches. La peau est également protégée par l'irradiation FLASH jusqu'à $32 \mathrm{~Gy}$, soit une dose presque double de celle qui produit une nécrose cutanée après une irradiation CONV. En revanche, l'irradiation FLASH s'est montrée aussi efficace que l'irradiation CONV sur les trois modèles tumoraux testés, et l'escalade de dose qu'autorise la réduction des complications au niveau des tissus sains a permis de parvenir à une cure complète dans deux cas sur trois.

L'irradiation FLASH a donc pour effet d'accroître le différentiel de réponse entre tumeurs et tissus sains. Ceci suggère que la méthode pourrait être utilisée, soit pour réduire l'incidence de complications, soit pour augmenter l'efficacité antitumorale.

\section{Des mécanismes aux applications cliniques}

Qu'est-ce qui peut expliquer un tel écart entre tumeurs et tissus sains? Une différence constante entre les deux types de tissus est que, chez l'adulte, les cellules des tissus sains se trouvent le plus souvent en état de quiescence (dormance), alors que la plupart des cellules tumorales se répliquent activement. Or, les voies de réparation des dommages de l'ADN dépendent pour une large part de la progression dans le cycle cellulaire et, selon les auteurs, le mécanisme de recombinaison homologue, qui seul permettrait une réparation fidèle des lésions complexes de l'ADN, est bloqué dans les cellules quiescentes $[9,10]$. Nous supposons donc que la différence entre les deux modalités d'irradiation s'explique par le fait que l'irradiation FLASH ne produit pas un sous-type de dommages dont la réparation nécessite la mise en œuvre de la recombinaison homologue. L'avenir dira si cette hypothèse se vérifie.

La radiothérapie FLASH est-elle transposable en thérapeutique humaine? Pour répondre à cette question il faut approfondir la connaissance des mécanismes biologiques qui sont à l'origine du phénomène que nous avons mis en évidence. D'autres limites sont d'ordre opérationnel et technologique. Ainsi, l'effet différentiel du FLASH a été obtenu avec un protocole extrêmement hypofractionné (une seule fraction). L'avantage sera-t-il conservé en fractionnant la dose? Quel sera alors l'étalement optimal: combien de fractions, quel intervalle? Les accélérateurs qui permettraient de générer des flux d'électrons et/ou de rayons- $X$ suffisamment élevés pour délivrer la radiothérapie en FLASH sont en cours de développement; cette tentative représente en soi un véritable défi technologique. En attendant, les faisceaux de PBS à haut débit de dose qui se mettent actuellement en place dans les centres de protonthérapie pourraient, au prix d'une modification des protocoles spatio-temporels de délivrance de la dose, permettre de déterminer si la radiothérapie FLASH apporte un bénéfice en situation clinique. $\varepsilon$ n tout état de cause, l'irradiation FLASH constitue un outil original et puissant pour étudier les paramètres biologiques fondamentaux qui différencient tumeurs et tissus sains du point de vue de la réponse au rayonnement. $\diamond$

\section{The radiotherapy FLASH to save healthy tissues}




\section{LIENS D'INTÉRÊT}

Les auteurs déclarent n'avoir aucun lien d'intérêt concernant les données publiées dans cet article.

\section{RÉFÉRENCES}

1. Prempree T, Michelsen A, Merz T. The repair time of chromosome breaks induced by pulsed $x$-rays on ultrahigh dose-rate. Int J Radiat Biol 1969 ; 15 : 571-4.

2. Schmid TE, Dollinger G, Hable V, et al. Relative biological effectiveness of pulsed and continuous 20 $\mathrm{MeV}$ protons for micronucleus induction in 3D human reconstructed skin tissue. Radiother Oncol 2010 ; 95 : 66-72.
3. Ponette V, Le Péchoux C, Deniaud-Alexandre $\varepsilon$, et al. Hyperfast, early cell response to ionizing radiation. Int J Radiat Biol 2000 ; 76 : 1233-43.

4. Fernet M, Ponette V, Deniaud-Alexandre $\varepsilon$, et al. Poly (ADP-ribose) polymerase, a major determinant of early cell response to ionizing radiation. Int J Radiat Biol 2000 ; 76 : 1621-9.

5. Sharplin J, Franko AJ. A quantitative histological study of strain-dependent differences in the effects of irradiation on mouse lung during the early phase. Radiat Res 1989 ; 119 : 1-14.

6. Noble PW, Barkauskas $C E$, Jiang D. Pulmonary fibrosis: patterns and perpetrators. The J Clin Invest 2012 $122: 2756-62$.

\section{NOUVELLE}

\section{Les bacilles de la tuberculose bovine \\ Une évolution aux dépens de la transmissibilité chez l'homme}

Roland Brosch ${ }^{1}$, Christophe Guilhot ${ }^{2}$
7. Favaudon V, Caplier L, Monceau V, et al. Ultrahigh dose-rate FLASH irradiation increases the differential response between normal and tumor tissue in mice. Sci Transl Med $2014 ; 6: 245 \mathrm{ra} 93$.

8. Yarnold J, Vozenin-Brotons MC. Pathogenetic mechanisms in radiation fibrosis. Radiother Oncol $2010 ; 97: 149-61$

9. Mohrin M, Bourke $\varepsilon$, Alexander D, et al. Hematopoietic stem cell quiescence promotes error-prone DNA repair and mutagenesis. Cell Stem Cell $2010 ; 7: 174-85$.

10. Blanpain C, Mohrin M, Sotiropoulou PA, Passegue $\varepsilon$. DNA-damage response in tissue-specific and cancer stem cells. Cell Stem Cell $2011 ; 8: 16-29$.
${ }^{1}$ Institut Pasteur, unité de pathogénomique mycobactérienne intégrée,

25 , rue du docteur Roux, 75015 Paris, France; ${ }^{2}$ CNRS, Université de Toulouse,

Université Paul Sabatier, Institut de pharmacologie et de biologie structurale, 205, route de Narbonne, 31000 Toulouse, France. roland.brosch@pasteur.fr christophe.guilhot@ipbs.fr

identifiés dès la fin du XIXe siècle, peu après la découverte par Robert Koch de MTB. Selon des documents historiques, les scientifiques de l'époque supposaient déjà des différences entre les bacilles tuberculeux d'origine humaine et bovine, malgré leurs ressemblances fortes $[3,4]$. Le modèle le plus communément admis était que MTB dérivait d'un bacille infectant les bovins. II a fallu attendre le début du $\mathrm{xxl}{ }^{\mathrm{e}}$ siècle et les avancées spectaculaires de la génomique comparative pour contredire ce modèle et établir un arbre phylogénétique clair des bacilles du complexe de MTB. Une cartographie très détaillée des différences génétiques a été construite, permettant d'établir un modèle évolutif fiable des bacilles tuberculeux $[5,6]$. Ce modèle place $M$. bovis à l'extrémité d'une lignée phylogénétique se séparant de la lignée des souches de MTB, et suggère que les souches animales dérivent d'un bacille humain (proche de M. africanum) [5-8], puis ont évolué vers une spécificité d'hôte $[9,10]$ (Figure 1).
1 Petit mammifère placentaire de l'odre des hyracoïde (Procavia capensis). II ressemble à une grosse marmotte, mais génétiquement, il serait plus proche des éléphants que des rongeurs. On le trouve en Égypte, au Liban, au Yémen et dans toute l'Afrique subsaharienne dans l'habitat des terres arides et pas dans la forêt équatoriale.

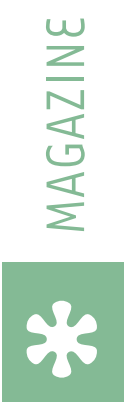

$>$ Il est fréquent d'observer une évolution Mycobacterium tuberculosis

La tuberculose est l'une des maladies infectieuses les plus dévastatrices dans du $x x l^{e}$ siècle, le nombre global de nouveaux cas de tuberculose par an est décès à 1,5 millions [1]. La tuberculose est provoquée par des bacilles, rassemMycobacterium tuberculosis (MTB) inclut les mycobactéries partageant plus de 99,9\% d'identité au niveau de leur 